\title{
Sum Perfect Square Labeling of Graphs
}

\author{
S.G. Sonchhatra \\ Assistant Prof. in Mathematics, \\ Govt. Eng. College, Rajkot, \\ Ph.D. Scholar, School Of Science, \\ R.K. University, Rajkot, Gujarat - INDIA. \\ sonchhabdasunil.20@gmail.com
}

\section{G. V. Ghodasara}

Assistant Prof. in Mathematics,

H. \& H. B. Kotak Institute of Science,

Rajkot, Gujarat - INDIA

gaurang_enjoy@yahoo.co.in

\begin{abstract}
Let $G=(V, E)$ be a $(p, q)$ graph and let $f: V(G) \rightarrow\{0,1,2, \ldots \ldots ., p-1\}$ be a bijection. We define $f^{*}: E(G) \rightarrow N$ by $f^{*}(u v)=[f(u)]^{2}+[f(v)]^{2}+2 f(u) \bullet f(v)$. If $f^{*}$ is injective, then $f$ is called sum perfect square labeling. A graph which admits sum perfect square labeling is called sum perfect square graph. Here we have focused on the graphs, whose edges can be labeled by a perfect square number only. In this paper we have initiated study of graphs which are sum perfect square.
\end{abstract}

Keywords: Sum perfect square graphs.

AMS Subject classification number: $05 \mathrm{C} 78$

\section{INTRODUCTION}

In our new defined labeling, the actual idea is to label all the edges of a graph by a perfect square number only. We consider simple, finite, undirected graph $G$ (with p vertices and q edges) throughout all this paper. The vertex set and edge set of graph $G$ are denoted by $V(G)$ and $E(G)$ respectively. In this paper $P_{n}$ denotes path with n vertices, $T_{m, n}$ denotes tadpole with $m+n$ vertices, $K_{1, n}$ denotes star graph with $\mathrm{n}+1$ vertices. For all other terminologies and notations we follow Harary[1].

Definition 1.1 : A chord of a cycle is an edge joining two non-adjacent vertices of cycle.

Definition 1.2: Two chords of a cycle are said to be twin chords if they form a triangle with an edge of the cycle. For positive integers $n$ and $p$ with $3 \leq p \leq n-2, C_{n, p}$ is the graph consisting of a cycle $C_{n}$ with a pair of twin chords with which the edges of $C_{n}$ form cycles $C_{p}, C_{3}$ and $C_{n+1-p}$ without chords.

Definition 1.3: The middle graph $M(G)$ of a graph $G$ is the graph whose vertex set is $V(G) \cup E(G)$ and in which two vertices are adjacent if and only if either they are adjacent edges of $G$ or one is a vertex of $G$ and other is an edge incident with it.

Definition 1.4: Let $G_{1}$ and $G_{2}$ be two graphs such that $V\left(G_{1}\right) \cap V\left(G_{2}\right)=\varnothing$. The join of $G_{1}$ and $G_{2}$ is denoted by $G_{1}+G_{2}$. It is the graph with $V\left(G_{1}+G_{2}\right)=V\left(G_{1}\right) \cup V\left(G_{2}\right)$ and $E\left(G_{1}+G_{2}\right)=E\left(G_{1}\right) \cup E\left(G_{2}\right) \cup J$, where $J=\left\{u v / u \in V\left(G_{1}\right)\right.$ and $\left.v \in V\left(G_{2}\right)\right\}$.

Definition 1.5: Let $G=(p, q)$ be a graph. A bijection $f: V(G) \rightarrow\{0,1,2, \ldots \ldots ., p-1\}$ is called sum perfect square labeling of $G$, if the induced function $f^{*}: E(G) \rightarrow N$ by $f *(u v)=[f(u)]^{2}+[f(v)]^{2}+2 f(u) \bullet f(v)$ is injective, for all $u, v \in V(G)$. A graph which admits sum perfect square labeling is called sum perfect square graph.

\section{LiTERATURE SURVEY}

V Ajitha, S. Arumugam and K. Germina[7] have initiated the study of Square Sum Graphs in 2009. Their work is closely related to Diophantine kinds of equations. The authors have shown several graphs which are Square sum. K. Germina et al[4] also proved several more graphs to be Square 
Sum graphs. J. Shiama [4] defined Square difference graphs and also find several graphs, which are Square difference. Above work motivates and leads us to define a new labeling, we say sum perfect square labeling. We say a graph to be sum perfect square if it admits sum perfect square labeling. Due to new defined labeling, we find the existence of the graphs, whose edges can be labeled by a perfect square number only.

\section{Main Results}

Lemma 3.1: $P_{n}$ is sum perfect square graph, for all $n \in N, n \geq 2$.

Proof: Let $\left\{v_{1}, v_{2}, \ldots \ldots, v_{n}\right\}$ be the successive vertices of path $P_{n}$. We define $f: V\left(P_{n}\right) \rightarrow\{0,1,2, \ldots ., n-1\}$ as $f\left(v_{i}\right)=i-1,1 \leq i \leq n$.

Injectivity: As function $f$ is increasing in terms of $i$, we get $f\left(v_{i}\right)<f\left(v_{i+1}\right)$ and so $f^{*}:(E(G)) \rightarrow N$ is injective. Hence $P_{n}$ is sum perfect square graph, for all $n \in N, n \geq 2$.

Lemma 3.2: Star $K_{1, n}$ is sum perfect square graph, for all $n \in N$.

Proof: Let $\left\{v_{0}, v_{1}, \ldots \ldots ., v_{n}\right\}$ be the successive vertices of star $K_{1, n}$, where $v_{0}$ is the apex vertex. We define $f: V\left(K_{1, n}\right) \rightarrow\{0,1,2, \ldots ., n\}$ as $f\left(v_{0}\right)=0$ and $f\left(v_{i}\right)=i, 1 \leq i \leq n$.

Injectivity: As function $f$ is increasing in terms of

$i$ for anyi, $m \in N$, with $i<m$, we have $f\left(v_{i}\right)<f\left(v_{m}\right)$, and so $f^{*}\left(v_{0} v_{i}\right)<f *\left(v_{0} v_{m}\right), 1 \leq i, m \leq n$.

So $f^{*}:(E(G)) \rightarrow N$ is injective. Hence $K_{1, n}$ is sum perfect square graph, for all $n \in N$.

Theorem 3.1: Every tree is sum perfect square graph.

Proof : Let $\left\{v_{0}, v_{1}, \ldots \ldots, v_{n}\right\}$ be the successive vertices of a tree $T$. Let us consider $T$ as a rooted tree. Let $v_{0}$ be the root vertex of $T$. Clearly $v_{0}$ is at vertex level 0 . Let $\left\{v_{1}, v_{2}, \ldots \ldots, v_{k}\right\}$ be the successive vertices at level $1, \quad 1 \leq k \leq n(k \in N) . \quad\left\{v_{k+1}, v_{k+2}, \ldots \ldots, v_{t}\right\}$ be the successive vertices at level 2 , $k+1 \leq t \leq n(t \in N)$ and so on. We define $f: V(T) \rightarrow\{0,1,2, \ldots ., n\}$ by $f\left(v_{i}\right)=i, 0 \leq i \leq n$.

Injectivity: At each consecutive level $l_{m}$ and $l_{m+1}, m \in N \cup\{0\}$, we get $f\left(v_{i}\right)<f\left(v_{j}\right)$,

for all $i$ and $j$, where vertex $v_{i}$ is at level $l_{m}$ and vertex $v_{j}$ is at level $l_{m+1}$. So $f^{*}:(E(T)) \rightarrow N$ is injective. Hence tree is sum perfect square graph.

An illustration of tree with 15 vertices is also provided below, for better understanding of defined labeling pattern.

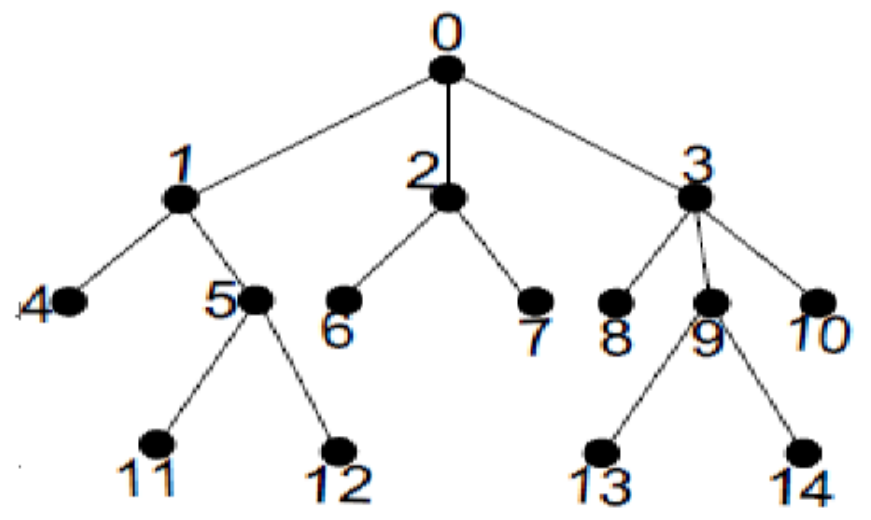

Theorem 3.2 : $C_{n}$ is sum perfect square graph, for all $n \geq 3$.

Proof : Let $\left\{v_{1}, v_{2}, \ldots \ldots, v_{n}\right\}$ be the successive vertices of cycle $C_{n}$. We define $f: V\left(C_{n}\right) \rightarrow\{0,1,2, \ldots ., n-1\}$ as follows: 
$f\left(v_{i}\right)=\left\{\begin{array}{l}\left.2 i-2, ; \quad 1 \leq i \leq \mid \frac{n}{2}\right\rceil \\ 2(n-i)+1 ; \quad\left\lceil\frac{n}{2}\right\rceil+1 \leq i \leq n\end{array}\right.$.

\section{Injectivity :}

For $1 \leq i \leq\left\lceil\frac{n}{2}\right\rceil$ since $f$ is increasing in terms of $i, f\left(v_{i}\right)<f\left(v_{i+1}\right)<f\left(v_{i+2}\right)$ and so we get $f *\left(v_{i} v_{i+1}\right)<f *\left(v_{i+1} v_{i+2}\right), 1 \leq i \leq\left\lceil\frac{n}{2}\right\rceil-1$. Moreover for $\left\lceil\frac{n}{2}\right\rceil+1 \leq i \leq n$, as $f$ is decreasing in terms of $i, f\left(v_{i}\right)>f\left(v_{i+1}\right)>f\left(v_{i+2}\right)$ and so we get $f *\left(v_{i} v_{i+1}\right)>f *\left(v_{i+1} v_{i+2}\right),\left\lceil\frac{n}{2}\right\rceil+1 \leq i \leq n$. We also note that $f *\left(v_{1} v_{n}\right)=1$, which is the smallest edge label in the graph.

Further $f *\left(v_{\left\lceil\frac{n}{2}\right\rceil} v_{\left\lceil\frac{n}{2}\right\rceil+1}\right)=\left\{\begin{array}{cc}(2 n-4)^{2}, & \text { if nis even, } \\ (2 n-3)^{2}, & \text { if nisodd }\end{array}\right.$ which is the highest edge label in the graph. Now $f\left(v_{t}\right)$ is even for each $t, 1 \leq t \leq\left\lceil\frac{n}{2}\right\rceil$ and $f\left(v_{k}\right)$ is odd for each $k,\left\lceil\frac{n}{2}\right\rceil+1 \leq k \leq n-1$, we get $f *\left(v_{t} v_{t+1}\right) \neq f *\left(v_{k} v_{k+1}\right)$. So $f^{*}:\left(E\left(C_{n}\right)\right) \rightarrow N$ is injective. Hence $C_{n}$ is sum perfect square graph, for all $n \geq 3$.

Theorem 3.3: Cycle $C_{n}$ with one chord is sum perfect square graph, for all $n \geq 4$.

Proof : Let $G$ be the cycle with one chord. Let $\left\{v_{1}, v_{2}, \ldots \ldots, v_{n}\right\}$ be the successive vertices of cycle $C_{n}$ and $e=v_{2} v_{n}$ be the chord of cycle $C_{n}$. The vertices $v_{1}, v_{2}$ and $v_{n}$ form a triangle in $C_{n}$ with chord $e$. We define $f: V\left(C_{n}\right) \rightarrow\{0,1,2, \ldots . ., n-1\}$ as follows:

$f\left(v_{i}\right)=\left\{\begin{array}{l}2 i-2, ; \quad 1 \leq i \leq\left\lceil\frac{n}{2}\right\rceil \\ 2(n-i)+1 ; \quad\left\lceil\frac{n}{2}\right\rceil+1 \leq i \leq n\end{array}\right.$.

\section{Injectivity:}

The chord $e=v_{2} v_{n}$ is labeled by 9, which is different from all other edge labels, as this edge label can be induced only by the vertex labels 0,3 and 1,2 , but after label the vertex $v_{1}$ by 1 and $v_{n}$ by 2 , the pair 0,3 will never meet each other. For $1 \leq i \leq\left\lceil\frac{n}{2}\right\rceil$, since $f$ is increasing in terms of $i, f\left(v_{i}\right)<f\left(v_{i+1}\right)<f\left(v_{i+2}\right)$ and so we get $f *\left(v_{i} v_{i+1}\right)<f *\left(v_{i+1} v_{i+2}\right), 1 \leq i \leq\left\lceil\frac{n}{2}\right\rceil-1$. Moreover for $\left\lceil\frac{n}{2}\right\rceil+1 \leq i \leq n, \quad$ as $f$ is decreasing in terms of $i, \quad f\left(v_{i}\right)>f\left(v_{i+1}\right)>f\left(v_{i+2}\right)$ and so we get $f *\left(v_{i} v_{i+1}\right)>f *\left(v_{i+1} v_{i+2}\right),\left\lceil\frac{n}{2}\right\rceil+1 \leq i \leq n . \quad$ Also $f *\left(v_{1} v_{n}\right)=1$, which is unique and smallest edge label among all the edge labels in the graph.

Further $f *\left(v_{\left\lceil\frac{n}{2}\right\rceil} v_{\left\lceil\frac{n}{2}\right\rceil+1}\right)=\left\{\begin{array}{ll}(2 n-4)^{2}, & \text { if nis even, } \\ (2 n-3)^{2}, & \text { if } n \text { is odd },\end{array}\right.$ which is the highest edge label and so different from all other edge labels in the graph. Now $f\left(v_{t}\right)$ is even for each $t, \quad 1 \leq t \leq\left\lceil\frac{n}{2}\right\rceil-1$ and $f\left(v_{k}\right)$ is odd for each $k,\left\lceil\frac{n}{2}\right\rceil+1 \leq k \leq n-1$, we get $f *\left(v_{t} v_{t+1}\right) \neq f *\left(v_{k} v_{k+1}\right)$. So all the edge labels are distinct. Hence $C_{n}$ with one chord is sum perfect square graph, for all $n \geq 4$. 
Theorem 3.4: Cycle $C_{n}$ with twin chords is sum perfect square graph, for all $n \geq 5$.

Proof : Let $G$ be the cycle with twin chords. Let $\left\{v_{1}, v_{2}, \ldots \ldots, v_{n}\right\}$ be the successive vertices of cycle $C_{n}$ and $e_{1}=v_{2} v_{n}$ and $e_{2}=v_{3} v_{n}$ be the two chords of cycle $C_{n}$.

We define $f: V\left(C_{n}\right) \rightarrow\{0,1,2, \ldots . ., n-1\}$ as follows:

$f\left(v_{i}\right)=\left\{\begin{array}{l}2 i-2, ; \quad 1 \leq i \leq\left\lceil\frac{n}{2}\right] \\ 2(n-i)+1 ; \quad\left[\frac{n}{2}\right]+1 \leq i \leq n\end{array}\right.$.

\section{Injectivity:}

The chord $e_{1}=v_{2} v_{n}$ is labeled by 9 , which is different from all other edge labels, as this edge label can be induced only by the vertex labels 0,3 and 1,2, but after label the vertex $v_{1}$ by 1 and $v_{n}$ by 2 , the pair 0,3 will never meet each other, similar argument is valid for $e_{2}=v_{3} v_{n}$, which is labeled by the label 25. For $1 \leq i \leq\left\lceil\frac{n}{2}\right\rceil$, since $f$ is increasing in terms of $i, f\left(v_{i}\right)<f\left(v_{i+1}\right)<f\left(v_{i+2}\right)$ and so we get $f *\left(v_{i} v_{i+1}\right)<f *\left(v_{i+1} v_{i+2}\right), 1 \leq i \leq\left\lceil\frac{n}{2}\right\rceil-1$. Moreover for $\left\lceil\left\lceil\frac{n}{2}\right\rceil+1 \leq i \leq n, \quad\right.$ as $f$ Is decreasing in terms of $i, \quad f\left(v_{i}\right)>f\left(v_{i+1}\right)>f\left(v_{i+2}\right)$ and $\quad$ so $\quad$ we $\quad$ get $f *\left(v_{i} v_{i+1}\right)>f *\left(v_{i+1} v_{i+2}\right),\left\lceil\frac{n}{2}\right\rceil+1 \leq i \leq n$. Also $f *\left(v_{1} v_{n}\right)=1$, which is unique and smallest edge label among all the edge labels in the graph.

Further $f^{*}\left(v_{\left\lceil\frac{n}{2}\right\rceil} v_{\left\lceil\frac{n}{2}\right\rceil+1}\right)=\left\{\begin{array}{ll}(2 n-4)^{2}, & \text { if nis even, } \\ (2 n-3)^{2}, & \text { if nis odd, }\end{array}\right.$ which is the highest edge label and so different from all other edge labels in the graph. Now $f\left(v_{t}\right)$ is even for each $t, \quad 1 \leq t \leq\left\lceil\frac{n}{2}\right\rceil-1$ and $f\left(v_{k}\right)$ is odd for each $k,\left\lceil\frac{n}{2}\right\rceil+1 \leq k \leq n-1$, we get $f *\left(v_{t} v_{t+1}\right) \neq f *\left(v_{k} v_{k+1}\right)$. So all the edge labels are distinct. Hence $C_{n}$ with twin chords is sum perfect square graph, for all $n \geq 5$.

$>$ Following figure justifies the labeling pattern, which we have defined in Theorem 2, 3 and 4.
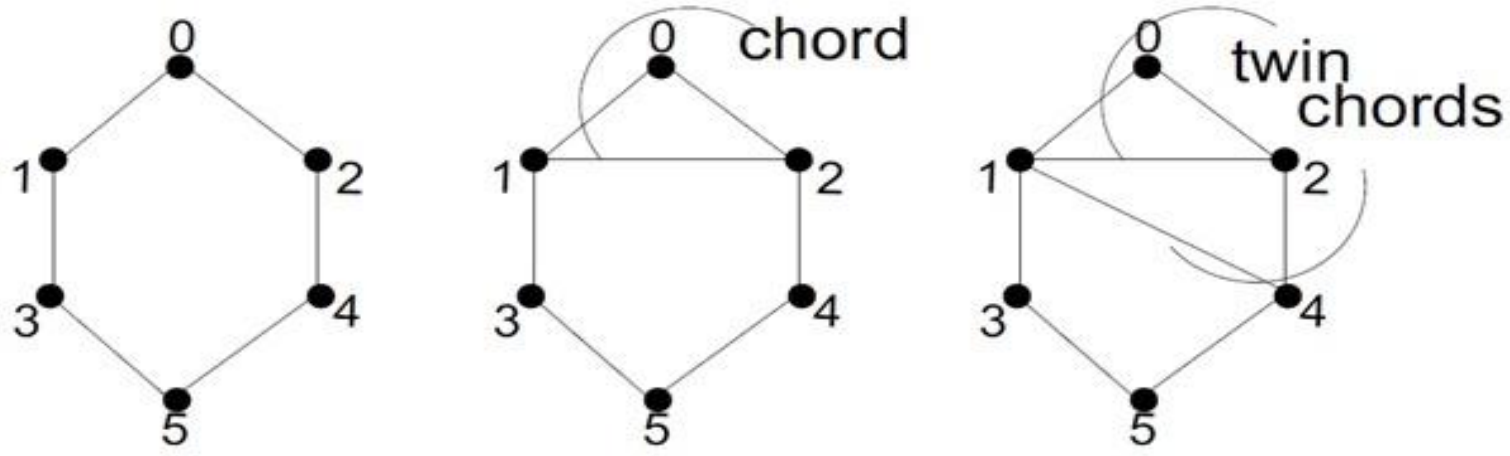

Theorem 3.5: $K_{n}$ is sum perfect square graph, for $n<4$.

Proof : For $n=1$ and 2, the graphs are special cases of tree and according to Theorem 3.1, $K_{1}$ and $K_{2}$ are sum perfect square graphs. For $n=3, K_{3}$ is the cycle $C_{3}$, and hence as per Theorem 3.2, $K_{3}$ is sum perfect square graph. For $n=4$, when we label the vertices of $K_{4}$, we come across the pair 1 , 2 and 0,3 , for which we get the same induced edge labels. Hence $K_{4}$ is not sum perfect square graph. For all $n \geq 4$, as $K_{4} \subset K_{5} \subset \ldots \ldots \ldots \ldots \subset K_{n}$, and so $K_{n}$ is not sum perfect square graph, for all $n \geq 4$. 
Theorem 3.6: Tadpole $T_{m, n}$ is sum perfect square gaph, for all positive integers $m$ and $n$.

Proof: Let $\left\{v_{1}, v_{2}, \ldots \ldots, v_{m}\right\}$ be the successive vertices of cycle $C_{m}$ and let $\left\{v_{m+1}, v_{m+2}, \ldots \ldots ., v_{m+n}\right\}$ be the successive vertices of path $P_{n}$ in tadpole $T_{m, n}$. Let $e=v_{m} v_{m+1}$ be the bridge in tadpole $T_{m, n}$, where $v_{m}$ is the vertex with the highest label corresponding to cycle $C_{n}$ and $v_{m+1}$ is the vertex with the smallest label corresponding to path $P_{n}$.

We define $f: V\left(T_{m, n}\right) \rightarrow\{0,1,2, \ldots . ., m+n-1\}$ as follows:

Case 1: $m$ is even.

$$
f\left(v_{i}\right)=\left\{\begin{array}{c}
m-2 i, 1 \leq i \leq\left\lceil\frac{m}{2}\right\rceil, \\
2 i-m-1,\left\lceil\frac{m}{2}\right\rceil+1 \leq i \leq m, \\
i-1, m+1 \leq i \leq m+n
\end{array}\right.
$$

\section{Injectivity:}

For $\quad 1 \leq i \leq\left\lceil\frac{m}{2}\right\rceil$, since $f$ is decreasing in terms of $i, f\left(v_{i}\right)>f\left(v_{i+1}\right)>f\left(v_{i+2}\right)$ and so we get $f *\left(v_{i} v_{i+1}\right)>f *\left(v_{i+1} v_{i+2}\right), 1 \leq i \leq\left\lceil\frac{m}{2}\right\rceil-1$. For $\left\lceil\frac{m}{2}\right\rceil+1 \leq i \leq m+n$, as $f$ is increasing in terms of $i, f\left(v_{i}\right)<f\left(v_{i+1}\right)<f\left(v_{i+2}\right)$ and so we get $f *\left(v_{i} v_{i+1}\right)<f *\left(v_{i+1} v_{i+2}\right),\left\lceil\frac{m}{2}\right\rceil+1 \leq i \leq m+n$. Moreover for Also $f^{*}\left(v_{\left\lceil\frac{m}{2}\right\rceil\left\lceil\frac{m}{2} v_{+1}\right.}\right)=1$, which is the smallest edge label among all the edge labels in this graph. Further $f^{*}\left(v_{1} v_{m}\right)=(2 m-3)^{2}$, is the highest edge label in cycle $C_{m}$ and according to the pattern which we defined, for $m+1 \leq i \leq m+n$, as $f$ is increasing in terms of $i, f\left(v_{i}\right)<f\left(v_{i+1}\right)<f\left(v_{i+2}\right)$ and so we get $f *\left(v_{i} v_{i+1}\right)<f *\left(v_{i+1} v_{i+2}\right)$. Now $f\left(v_{t}\right)$ is even for each $t, \quad 1 \leq t \leq\left\lceil\frac{m}{2}\right\rceil$ and $f\left(v_{k}\right)$ is odd for each $k,\left\lceil\frac{m}{2}\right\rceil+1 \leq k \leq m$, we get $f *\left(v_{t} v_{t+1}\right) \neq f *\left(v_{k} v_{k+1}\right)$. So all the edge labels are distinct. Hence $f^{*}:\left(E\left(T_{m, n}\right)\right) \rightarrow N$ is injective in this case.

Case 2: $m$ is odd.

$$
f\left(v_{i}\right)=\left\{\begin{array}{c}
m-2 i-1,1 \leq i \leq\left\lfloor\frac{m}{2}\right\rfloor, \\
2 i-m-1,\left\lfloor\frac{m}{2}\right\rfloor+1 \leq i \leq m-1, \\
i-1, m+1 \leq i \leq m+n
\end{array}\right.
$$




\section{Injectivity:}

For $1 \leq i \leq\left\lfloor\frac{m}{2}\right\rfloor$, since $f$ is decreasing in terms of $i, \quad f\left(v_{i}\right)>f\left(v_{i+1}\right)>f\left(v_{i+2}\right)$ and so we get $f *\left(v_{i} v_{i+1}\right)>f *\left(v_{i+1} v_{i+2}\right), 1 \leq i \leq\left\lfloor\frac{m}{2}\right\rfloor-1$. For $\left\lfloor\frac{m}{2}\right\rfloor+1 \leq i \leq m+n$, as $f$ is increasing in terms of $i, f\left(v_{i}\right)<f\left(v_{i+1}\right)<f\left(v_{i+2}\right)$ and so we get $f *\left(v_{i} v_{i+1}\right)<f *\left(v_{i+1} v_{i+2}\right),\left\lfloor\frac{m}{2}\right\rfloor+1 \leq i \leq m+n$. Moreover for Also $f *\left(v_{\left[\frac{m}{2}\right\rfloor} v_{\left\lfloor\frac{m}{2}\right\rfloor+1}\right)=1$, which is the smallest edge label among all the edge labels in this graph. Further $f^{*}\left(v_{1} v_{m}\right)=(2 m-3)^{2}$, is the highest edge label in cycle $C_{m}$ and according to the pattern which we defined, for $m+1 \leq i \leq m+n$, as $f$ is increasing in terms of $i, f\left(v_{i}\right)<f\left(v_{i+1}\right)<f\left(v_{i+2}\right)$ and so we get $f^{*}\left(v_{i} v_{i+1}\right)<f^{*}\left(v_{i+1} v_{i+2}\right)$. Now $f\left(v_{t}\right)$ is even for each $t, \quad 1 \leq t \leq\left\lfloor\frac{m}{2}\right\rfloor$ and $f\left(v_{k}\right)$ is odd for each $k,\left\lfloor\frac{m}{2}\right\rfloor+1 \leq k \leq m$, we get $f^{*}\left(v_{t} v_{t+1}\right) \neq f *\left(v_{k} v_{k+1}\right)$. So all the edge labels are distinct. Hence $f^{*}:\left(E\left(T_{m, n}\right)\right) \rightarrow N$ is injective in this case. Tadpole $T_{m, n}$ is sum perfect square graph, for all positive integers $m$ and $n$.

$>$ The following figure indicates the labelling pattern defined in Theorem no. 6

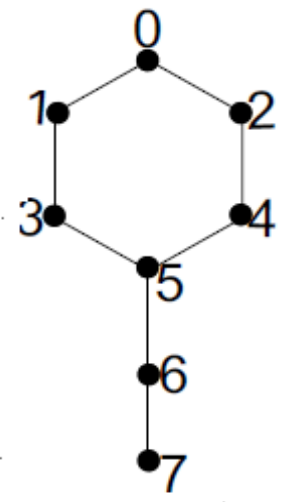

\section{CONJECTURE}

Every odd graph (i.e. all the vertices are having odd degree) $G$, in which all the vertices $v_{i}$ are such that $d\left(v_{i}\right) \geq 3$ then $G$ is not a sum perfect square graph.

\section{Conclusion}

The labeling introduced in this paper will open new notion of study of some families of several graphs, in which all the edges must be labeled by a distinct perfect square integer. Related to new introduced labeling, several new theorems have been proved in this paper. At the end a conjecture have been put, which can be treated as an open problem.

\section{ACKNOWLEDGEMENT}

The authors are thankful to anonymous referee for their valuable comments and suggestions.

\section{REFERENCES}

[1] Harary F., Graph Theory, Addision Wesley, Reading, MA, 1969.

[2] Gallian J.A., A dynamic survey of graph labeling, The Electronics Journal of Combinatorics, 17(2014), \#DS6 1-250.

[3] Cahit I., Cordial Graphs: A Weaker Version of Graceful and Harmonic Graphs, Ars Combinatoria, 23(1987), 201-207. 
[4] Germina K.A., Sebastian R., Further Results on Square Sum Graphs, International Mathematical Forum, 8(2013), 47-57.

[5] Shiama J., Some Special Types of Square Difference Graphs, International Journal Of Mathematical Archieve, 3(2012), 2369-2374

[6] Shiama J., Square Difference Labeling for Some Graphs, International Journal Of Computer Applications, 44(2012), 6523-8399.

[7] Ajitha V., Arumugam S., Germina K., On Square Sum Graphs, AKCE Journal of Graphs and Combinatorics, 1(2009), 1-10.

\section{AUTHORS' BIOGRAPHY}

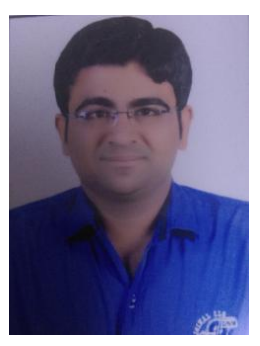

Mr. Sunil G. Sonchhatra, received his M.Sc., B.Ed. and M.Phil degrees from Saurashtra University, Rajkot in year 2007, 2008 and 2010 respectively. Recently he is working as an assistant professor in Mathematics at Government Engineering College, Rajkot and pursuing his Ph.D. from R.K. University, Rajkot, Gujarat, India.

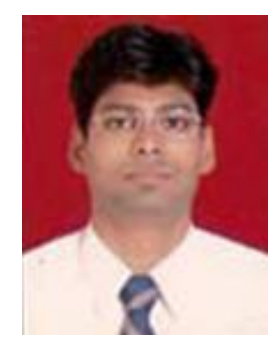

Dr. Gaurang V. Ghodasara, received his M.Sc.degree from S.P. University, Vallabh Vidyanagar(Gujarat). He also received his M.Phil and Ph.D. degrees from Saurashtra University, Rajkot (Gujarat) in year 2005 and 2009 respectively. He has worked as an assistant professor in Mathematics at Atmiya Institute of Technology \& Science, Rajkot and L.E. College, Morbi.

Recently he is working as an assistant professor in Mathematics Department at H.\& H.B. Kotak Institute of Science, Rajkot(Gujarat). He is registered guide at R.K. University, Rajkot. 